\title{
THE RADON TRANSFORM OF BOEHMIANS
}

\author{
PIOTR MIKUSIŃSKI AND AHMED ZAYED
}

(Communicated by Andrew M. Bruckner)

\begin{abstract}
The Radon transform, which enables one to reconstruct a function of $N$ variables from the knowledge of its integrals over all hyperplanes of dimension $N-1$, has been extended to Schwartz distributions by several people including Gelfand, Graev, and Vilenkin, who extended it to tempered distributions.

In this paper we extend the Radon transform to a space of Boehmians. Boehmians are defined as sequences of convolution quotients and include Schwartz distributions and regular Mikusiński operators.

Our extension of the Radon transform includes generalized functions of infinite order with compact support. The technique used in this paper is based on algebraic properties of the Radon transform and its convolution structure rather than on their analytic properties. Our results do not contain nor are contained in those obtained by Gelfand et al.
\end{abstract}

\section{INTRODUCTION}

The Radon transform, which was defined and studied by Radon [11] in 1917, has emerged in the last two decades as one of the most important mathematical tools in various fields of applications in physics, engineering, astronomy, and medicine. The applications of the Radon transform are numerous; however, the most widely known is probably its application in medical imaging, where it plays an essential role in the field of Computerized Axial Tomography or Computerized Assisted Tomography, commonly known to the public as CAT scans. The importance of the Radon transform and its inverse lies in the fact that they solve the following reconstruction problem: construct a function $f(x, y)$ given that its integrals over all straight lines are known; or more generally, construct a function $f\left(x_{1}, \ldots, x_{N}\right)$ given that its integrals over all hyperplanes of dimension $N-1$ are known. To see how this simply stated reconstruction problem plays a central role in a wide range of applications, we refer the reader to [2, Chapter 1] for an excellent exposition.

Although in most applications the function $f$ is assumed to have compact support, in theory it is usually assumed to be in the Schwartz space $\mathscr{S}\left(\mathbb{R}^{N}\right)$ $(N \geq 2)$ of infinitely differentiable rapidly decreasing functions. The extension

Received by the editors October 20, 1991.

1991 Mathematics Subject Classification. Primary 44A12, 44A40; Secondary 46F12, 92C55.

Key words and phrases. Radon transform, Boehmians, generalized functions. 
of the Radon transform to generalized functions has been studied by many people $[5,6]$, including Gelfand, Graev, and Vilenkin [4], who used the method of the adjoint transform to define the Radon transform of a tempered distribution $F$, i.e., $F \in \mathscr{S}^{\prime}$. Denote by $S^{N-1}$ the unit sphere in $\mathbb{R}^{N}$ and by $\Sigma^{N}$ the generalized cylinder $\mathbb{R} \times S^{N-1}$. We recall that if $f \in \mathscr{S}\left(\mathbb{R}^{N}\right)$, then its Radon transform $\hat{f}(p, \boldsymbol{\xi})$, where $(p, \boldsymbol{\xi})$ is a point on $\Sigma^{N}$, has the following properties:

(1) $\hat{f}(p, \boldsymbol{\xi})$ is an even homogeneous function of degree -1 , i.e., $\hat{f}(\alpha p, \alpha \boldsymbol{\xi})$ $=\hat{f}(p, \boldsymbol{\xi}) /|\alpha|, \alpha \neq 0$.

(2) $\hat{f}(p, \xi)$ is infinitely differentiable with respect to $\boldsymbol{\xi}$ and with respect to $p$ for $\boldsymbol{\xi} \neq 0$.

(3) $\hat{f}(p, \xi)$ and all its derivatives with respect to $p$ and $\boldsymbol{\xi}$ are of order $o\left(1 /|p|^{k}\right)$ as $|p| \rightarrow \infty$ for all $k$ and uniformly in $\xi$.

(4) For any nonnegative integer $k$, the integral $\int_{\mathbb{R}} \hat{f}(p, \boldsymbol{\xi}) p^{k} d p$ is a polynomial in $\boldsymbol{\xi}$, homogeneous of degree $k$.

Let $\psi(p, \boldsymbol{\xi})=\partial^{N-1} \hat{f}(p, \xi) / \partial p^{N-1}$, where $\hat{f}$ satisfies (1)-(4). Provided with an appropriate topology, the linear space of all such $\psi$ 's becomes a testingfunction space and will be denoted by $\widehat{\mathscr{S}_{\Sigma}}[4,14]$. Thus, according to Gelfand, Graev, and Vilenkin, the Radon transform $\widehat{F}$ of a tempered distribution $F$ is defined as a continuous linear functional on $\widehat{\mathscr{S}_{\Sigma}}$ given by

$$
\langle F, f\rangle=C_{N}\langle\widehat{F}, \psi\rangle,
$$

where $C_{N}$ is a constant depending only on the dimension $N$. The space of all continuous linear functionals on $\widehat{\mathscr{S}_{\Sigma}}$ is denoted $\widehat{\mathscr{S}_{\Sigma}^{\prime}}$.

The theory of distributions and generalized functions as developed by Schwartz [13], Gelfand and Shilov [3], and later by Zemanian [14], regards generalized functions as elements of the dual space of some space of testing functions. Following a different approach to generalized functions, Mikusiński [7] developed his theory of the Mikusinski operators in which a generalized function is regarded as an equivalence class of convolution quotients. Later, extending an idea of regular operators introduced by Boehme [1], J. Mikusiński and P. Mikusiński [8] constructed a larger class of mathematical objects, commonly known as Boehmians, which embraces regular operators, Schwartz distributions, and other spaces of generalized functions [10]. A Boehmian is defined as an equivalence class of "quotients of sequences."

The aim of this paper is to extend the Radon transform to a subclass of Boehmians, called integrable Boehmians. Our approach is different from that used by Helgason [5] and by Gelfand, Graev, and Vilenkin [4], and our results neither contain nor are contained in theirs.

\section{BOEHMIANS}

Let $G$ be a complex linear space and let $H$ be a subspace of $G$. Let $\otimes$ be a binary operation from $G \times H$ into $G$ such that the following conditions are satisfied:

(2.1) If $\phi, \psi \in H$, then $\phi \otimes \psi \in H$ and $\phi \otimes \psi=\psi \otimes \phi$.

(2.2) If $f \in G$ and $\phi, \psi \in H$, then $(f \otimes \phi) \otimes \psi=f \otimes(\phi \otimes \psi)$. 
(2.3) If $f, g \in G, \phi \in H$, and $\lambda \in \mathbb{C}$, then $(f+g) \otimes \phi=f \otimes \phi+g \otimes \phi$ and $\lambda(f \otimes \phi)=(\lambda f \otimes \phi)$.

Let $\Delta$ be a family of sequences of elements of $H$ such that

(2.4) If $f \in G,\left\{\phi_{n}\right\} \in \Delta$, and $f \otimes \phi_{n}=0$ for all $n \in \mathbb{N}$, then $f=0$.

(2.5) If $\left\{\phi_{n}\right\},\left\{\psi_{n}\right\} \in \Delta$, then $\left\{\phi_{n} \otimes \psi_{n}\right\} \in \Delta$.

A pair of sequences $\left(f_{n}, \phi_{n}\right)$ is called a quotient of sequences and is denoted by $\left(f_{n}\right) /\left(\phi_{n}\right)$ if $f_{n} \in G$ for $n=1,2, \ldots,\left\{\phi_{n}\right\} \in \Delta$ and $f_{n} \otimes \phi_{m}=f_{m} \otimes \phi_{n}$ for all $m, n \in \mathbb{N}$. Two quotients of sequences $\left(f_{n}\right) /\left(\phi_{n}\right)$, and $\left(g_{n}\right) /\left(\psi_{n}\right)$ are equivalent if $f_{n} \otimes \psi_{n}=g_{n} \otimes \phi_{n}$ for every $n \in \mathbb{N}$. The equivalence class of $\left(f_{n}\right) /\left(\phi_{n}\right)$ will be denoted by $\left[f_{n} / \phi_{n}\right]$. The space of equivalence classes of quotients of sequences will be denoted by $\mathfrak{B}(G, H, \otimes, \Delta)$ and its elements will be called Boehmians.

Addition and multiplication by scalars are defined in $\mathfrak{B}(G, H, \otimes, \Delta)$ as

$$
\begin{gathered}
\lambda\left[f_{n} / \phi_{n}\right]=\left[\lambda f_{n} / \phi_{n}\right], \\
{\left[f_{n} / \phi_{n}\right]+\left[g_{n} / \psi_{n}\right]=\left[\left(f_{n} \otimes \psi_{n}+g_{n} \otimes \phi_{n}\right) / \phi_{n} \otimes \psi_{n}\right] .}
\end{gathered}
$$

Then $\mathfrak{B}(G, H, \otimes, \Delta)$ is a vector space. The mapping $f \mapsto\left[f \otimes \phi_{n} / \phi_{n}\right]$ is an isomorphism of $G$ with a subspace of $\mathfrak{B}(G, H, \otimes, \Delta)$. It is convenient to treat $G$ as a subspace of $\mathfrak{B}(G, H, \otimes, \Delta)$.

If $F=\left[f_{n} / \phi_{n}\right] \in \mathfrak{B}(G, H, \otimes, \Delta)$ and $F^{\prime}=\left[f_{n}^{\prime} / \psi_{n}\right] \in \mathfrak{B}(H, H, \otimes, \Delta)$, then we can define $F \otimes F^{\prime}$ as

$$
F \otimes F^{\prime}=\left[f_{n} \otimes f_{n}^{\prime} / \psi_{n} \otimes \phi_{n}\right] .
$$

It is easy to see that $F \otimes F^{\prime} \in \mathfrak{B}(G, H, \otimes, \Delta)$ and that it is an extension of $\otimes$ from $G \otimes H$ onto $\mathfrak{B}(G, H, \otimes, \Delta) \times \mathfrak{B}(H, H, \otimes, \Delta)$. Using the identification of $H$ with a subspace of $\mathfrak{B}(H, H, \otimes, \Delta)$ we can write

$$
\left[f_{n} / \phi_{n}\right] \otimes \psi=\left[f_{n} \otimes \psi / \phi_{n}\right]
$$

and, in particular, $\left[f_{n} / \phi_{n}\right] \otimes \phi_{n}=f_{n}$.

If $G$ is equipped with a notion of convergence, then we can define a convergence in $\mathfrak{B}$. We say that a sequence of Boehmians $\left\{F_{n}\right\}$ is $\Delta$-convergent to a Boehmian $F$, and we write $\Delta$ - $\lim _{n \rightarrow \infty} F_{n}=F$ if there exists $\left\{\delta_{n}\right\} \in \Delta$ such that $\left(F_{n}-F\right) \otimes \delta_{n} \in G$ for every $n \in \mathbb{N}$ and the sequence $\left\{\left(F_{n}-F\right) \otimes \delta_{n}\right\}$ converges to zero in $G$. It can be proved that, under certain conditions, $\mathfrak{B}$ equipped with $\Delta$-convergence is a complete quasi-normed space (see [9]).

In this paper we are going to use four different spaces of Boehmians.

By $\mathscr{L}^{1}\left(\mathbb{R}^{N}\right)$ we denote the space of complex-valued Lebesgue integrable functions on $\mathbb{R}^{N}$ and by $\mathscr{D}\left(\mathbb{R}^{N}\right)$ the space of complex-valued infinitely differentiable functions with compact support. If $f \in \mathscr{L}^{1}\left(\mathbb{R}^{N}\right)$ and $\phi \in \mathscr{D}\left(\mathbb{R}^{N}\right)$, then by $f * g$ we mean the convolution product of $f$ and $\phi$, i.e.,

$$
(f * \phi)(\mathbf{y})=\int_{\mathbb{R}^{N}} f(\mathbf{x}) \phi(\mathbf{y}-\mathbf{x}) d \mathbf{x},
$$

where $\mathbf{x}=\left(x_{1}, \ldots, x_{N}\right) \in \mathbb{R}^{N}$ and $d \mathbf{x}=d x_{1} \cdots d x_{N}$.

For $\phi \in \mathscr{D}\left(\mathbb{R}^{N}\right)$, define $s(\phi)$ to be the radius of the smallest closed ball at the origin that contains the support of $\phi$, i.e.,

$$
s(\phi)=\inf \left\{\varepsilon>0: \operatorname{supp} \phi \subset B_{\varepsilon}\right\}
$$


where $\|\mathbf{x}\|=\left\|\left(x_{1}, \ldots, x_{N}\right)\right\|=\left(x_{1}^{2}+\cdots+x_{N}^{2}\right)^{1 / 2}$ and $B_{\varepsilon}=\left\{\mathbf{x} \in \mathbb{R}^{N}:\|\mathbf{x}\|<\varepsilon\right\}$.

A sequence of real-valued functions $\delta_{1}, \delta_{2}, \cdots \in \mathscr{D}\left(\mathbb{R}^{N}\right)$ is called a delta sequence if

(2.6) $\int_{\mathbb{R}^{N}} \delta_{n}(\mathbf{x}) d \mathbf{x}=1$ for every $n \in \mathbb{N}$,

(2.7) $\int_{\mathbb{R}^{N}}\left|\delta_{n}(\mathbf{x})\right| d \mathbf{x}<M$ for some $M>0$ and all $n \in \mathbb{N}$,

(2.8) $s\left(\delta_{n}\right) \rightarrow 0$ as $n \rightarrow \infty$.

The collection of all delta sequences will be denoted by $\Delta_{0}$. It is easy to check that, for $G=\mathscr{L}^{1}\left(\mathbb{R}^{N}\right), H=\mathscr{D}\left(\mathbb{R}^{N}\right), \otimes=*$, and $\Delta=\Delta_{0}$, conditions (2.1)-(2.5) are satisfied. The corresponding space of Boehmians will be denoted by $\mathfrak{B}_{\mathscr{L}}$, i.e.,

$$
\mathfrak{B}_{\mathscr{L}}=\mathfrak{B}\left(\mathscr{L}^{1}\left(\mathbb{R}^{N}\right), \mathscr{D}\left(\mathbb{R}^{N}\right), *, \Delta_{0}\right) .
$$

Elements of $\mathfrak{B}_{\mathscr{L}}$ are called integrable Boehmians.

By $\mathscr{S}\left(\mathbb{R}^{N}\right)$ we denote the space of complex-valued infinitely differentiable rapidly decreasing functions on $\mathbb{R}^{N}$. More precisely, an infinitely differentiable function $f$ on $\mathbb{R}^{N}$ is in $\mathscr{S}\left(\mathbb{R}^{N}\right)$ if and only if

$$
\|f\|_{m}=\sup _{|\alpha| \leq m} \sup _{\mathbf{x} \in \mathbb{R}^{N}}\left(1+\|\mathbf{x}\|^{2}\right)^{m}\left|\partial^{|\alpha|} f / \partial \mathbf{x}^{\alpha}\right|<\infty,
$$

where $\alpha=\left(\alpha_{1}, \ldots, \alpha_{N}\right),|\alpha|=\alpha_{1}+\cdots+\alpha_{N}$, and

$$
\partial^{|\alpha|} / \partial \mathbf{x}^{\alpha}=\partial^{|\alpha|} /\left(\partial x_{1}^{\alpha_{1}} \cdots \partial x_{N}^{\alpha_{N}}\right) .
$$

Now, for $G=H=\mathscr{S}\left(\mathbb{R}^{N}\right), \otimes=*$, and $\Delta=\Delta_{0}$, conditions (2.1)-(2.5) are satisfied. The corresponding space of Boehmians will be denoted by $\mathfrak{B}_{\mathscr{S}}$, i.e.,

$$
\mathfrak{B}_{\mathscr{S}}=\mathfrak{B}\left(\mathscr{S}\left(\mathbb{R}^{N}\right), \mathscr{S}\left(\mathbb{R}^{N}\right), *, \Delta_{0}\right) .
$$

Elements of $\mathfrak{B}_{\mathscr{L}}$ are called rapidly decreasing Boehmians.

Note that $\mathfrak{B}_{\mathscr{S}} \subset \mathfrak{B}_{\mathscr{L}}$. Moreover, every Boehmian $F \in \mathfrak{B}_{\mathscr{L}}$ has a representation $\left[f_{n} / \phi_{n}\right]$ such that $f_{n} \in \mathscr{C}^{\infty}\left(\mathbb{R}^{N}\right)$ for all $n \in \mathbb{N} ; \mathscr{C}^{\infty}\left(\mathbb{R}^{N}\right)$ denotes the space of infinitely differentiable functions. Indeed, if $F=\left[g_{n} / \psi_{n}\right]$ is any representation and $\left\{\delta_{n}\right\} \in \Delta_{0}$, then the representation $F=\left[g_{n} * \delta_{n} / \psi_{n} * \delta_{n}\right]$ has the desired property. Using such a representation we can define derivatives of a Boehmian $F$ by

$$
\frac{\partial^{|\alpha|} F}{\partial \mathbf{x}^{\alpha}}=\left[\frac{\partial^{|\alpha|} f_{n}}{\partial \mathbf{x}^{\alpha}} / \phi_{n}\right] .
$$

The defined operation has the usual properties and agrees with the usual differentiation if $F$ corresponds to a differentiable function.

Now we are going to define two spaces of Boehmians on $\Sigma^{N}$. Let $f$ and $g$ be functions on $\Sigma^{N}$. By $f \odot g$ we denote the convolution of $f$ and $g$ with respect to $p$, i.e.,

$$
(f \odot g)(p, \boldsymbol{\xi})=\int_{\mathbb{R}} f(t, \boldsymbol{\xi}) g(p-t, \boldsymbol{\xi}) d t .
$$

Thus, if $f \odot g$ exists, it is a function on $\Sigma^{N}$.

Let $\mathscr{L}\left(\Sigma^{N}\right)$ denote the space of all complex-valued functions $f(p, \xi)$ on $\Sigma^{N}$ such that $f(\cdot, \xi)$ is Lebesgue integrable for almost all $\boldsymbol{\xi} \in S^{N-1}$. Let $\mathscr{D}\left(\Sigma^{N}\right)$ be the space of all infinitely differentiable functions on $\Sigma^{N}$ with compact support. Let $\Delta_{\Sigma}$ denote the family of all sequences $\phi_{1}, \phi_{2}, \ldots \in \mathscr{D}\left(\Sigma^{N}\right)$ such that

(2.10) $\int_{\mathbb{R}} \phi_{n}(p, \boldsymbol{\xi}) d p=1$ for every $\boldsymbol{\xi} \in S^{N-1}$ and $n \in \mathbb{N}$, 
(2.11) $\sup _{\boldsymbol{\xi} \in S^{N-1}} \int_{\mathbb{R}}\left|\phi_{n}(p, \xi)\right| d p<M$ for some $M>0$ and all $n \in \mathbb{N}$,

(2.12) $\sup _{\boldsymbol{\xi} \in S^{N-1}} s\left(\phi_{n}(\cdot, \boldsymbol{\xi})\right) \rightarrow 0$ as $n \rightarrow \infty$.

It is easy to check that $\Delta_{\Sigma}$ satisfies conditions (2.4) and (2.5).

Let

$$
\mathfrak{B}_{\mathscr{L}(\Sigma)}=\mathfrak{B}\left(\mathscr{L}\left(\Sigma^{N}\right), \mathscr{D}\left(\Sigma^{N}\right), \odot, \Delta_{\Sigma}\right)
$$

and

$$
\mathfrak{B}_{\mathscr{S}(\Sigma)}=\mathfrak{B}\left(\mathscr{S}\left(\Sigma^{N}\right), \mathscr{D}\left(\Sigma^{N}\right), \odot, \Delta_{\Sigma}\right)
$$

where $\mathscr{S}\left(\Sigma^{N}\right)$ denote the space of all complex-valued infinitely differentiable functions $f$ on $\Sigma^{N}$ such that

$$
\|f\|_{\Sigma, m}=\sup _{\boldsymbol{\xi} \in S^{N-1}} \sup _{k \leq m} \sup _{p \in \mathbb{R}}\left(1+p^{2}\right)^{m}\left|\frac{\partial^{k}}{\partial p^{k}} f(p, \boldsymbol{\xi})\right|<\infty .
$$

Elements of $\mathfrak{B}_{\mathscr{L}(\Sigma)}$ and $\mathfrak{B}_{\mathscr{S}(\Sigma)}$ are called integrable Boehmians and rapidly decreasing Boehmians on $\Sigma^{N}$, respectively.

If the convergence in $\mathscr{S}\left(\mathbb{R}^{N}\right)$ is defined by the seminorms in (2.9), then $\mathfrak{B}_{\mathscr{S}}=\mathfrak{B}\left(\mathscr{S}\left(\mathbb{R}^{N}\right), \mathscr{S}\left(\mathbb{R}^{N}\right), *, \Delta_{0}\right)$ with $\Delta$-convergence is a complete quasinormed space. Moreover, addition, multiplication by scalars, and convolution with functions from $\mathscr{D}\left(\mathbb{R}^{N}\right)$ are continuous operations. Similarly, if the convergence in $\mathscr{S}\left(\Sigma^{N}\right)$ is defined by the seminorms in $(2.13)$, then $\mathfrak{B}_{\mathscr{S}(\Sigma)}=$ $\mathfrak{B}\left(\mathscr{L}\left(\Sigma^{N}\right), \mathscr{D}\left(\Sigma^{N}\right), \odot, \Delta_{\Sigma}\right)$ is a complete quasi-normed space.

\section{THE RADON TRANSFORM}

The Radon transform $\check{f}$ or $\mathscr{R} f$ of a function of two variables $f(x, y)$ is defined by

$$
\check{f}=\mathscr{R} f=\int_{L} f(x, y) d s
$$

whenever the integral exists, where $L$ runs over all possible straight lines in the $x y$-plane and $d s$ is an increment of length along $L$. To be more precise, for a fixed straight line $L$, the integral (3.1) is called a "slice" or a "sample" of the Radon transform and the totality of all these samples constitutes the Radon transform of $f$. Since the equation of any straight line in the $x y$-plane can be written in the form $p=x \cos \phi+y \sin \phi=\mathbf{x} \cdot \boldsymbol{\xi}$, where $\mathbf{x}=(x, y)$, $\xi=(\cos \phi, \sin \phi)$, and $0 \leq \phi \leq 2 \pi$, it is not hard to see [2] that (3.1) can be put in the form

$$
\check{f}(p, \boldsymbol{\xi})=\int_{\mathbb{R}^{2}} f(\mathbf{x}) \boldsymbol{\delta}(p-\mathbf{x} \cdot \boldsymbol{\xi}) d \mathbf{x},
$$

where $d \mathbf{x}=d x d y$ and $\delta$ is the Dirac delta distribution.

It is easy to see that if $f$ is integrable over $\mathbb{R}^{2}$ then $\breve{f}(p, \xi)$ exists for almost all $p$ and $\xi$.

The last form of the Radon transform enables us to extend it to higher dimensions as follows. Let $\xi$ denote a unit vector in $\mathbb{R}^{N}$. Then, since the equation of any hyperplane in $\mathbb{R}^{N}$ can be written in the form $p=\mathbf{x} \cdot \boldsymbol{\xi}$, we define the $N$-dimensional Radon transform of a function of $N$ variables $f(\mathbf{x})$ as

$$
\check{f}(p, \boldsymbol{\xi})=(\mathscr{R} f)(p, \boldsymbol{\xi})=\int_{\mathbb{R}^{N}} f(\mathbf{x}) \delta(p-\mathbf{x} \cdot \boldsymbol{\xi}) d \mathbf{x} .
$$


Since $\xi$ can be regarded as a point on the generalized sphere $S^{N-1}, \check{f}$ can be viewed as a function defined on the generalized cylinder $\Sigma^{N}=\mathbb{R} \times S^{N-1}$. For more details on the Radon transform we refer the reader to [2, 4, 5].

The following properties of the Radon transform are easy to establish:

(3.4) $\check{f}(\alpha p, \alpha \boldsymbol{\xi})=\check{f}(p, \xi) /|\alpha|$ for any real $\alpha \neq 0$.

(3.5) $\mathscr{R}(f(A \mathbf{x}))=|\operatorname{det} B| \check{f}\left(p, B^{\mathrm{T}} \boldsymbol{\xi}\right)$ where $B=A^{-1}$.

(3.6) $\mathscr{R}(f(\mathbf{x}+\mathbf{a}))=\check{f}(p+\boldsymbol{\xi} \cdot \mathbf{a}, \boldsymbol{\xi})$.

(3.7) $\check{f}(p, \xi)=\int_{\mathbb{R}} \tilde{f}(\omega \xi) e^{2 \pi i \omega p} d \omega$, where $\tilde{f}$ denotes the Fourier transform of $f$ :

$$
\tilde{f}(\mathbf{y})=\int_{\mathbb{R}^{N}} f(\mathbf{x}) e^{-2 \pi i \mathbf{x} \cdot \mathbf{y}} d \mathbf{x} .
$$

It is proven in [2, Chapter 5] that the original function $f$ can be recovered from its Radon transform according to the formulas

$$
f(\mathbf{x})=\frac{1}{(2 \pi i)^{N}} \int_{|\boldsymbol{\xi}|=1} d \boldsymbol{\xi} \int_{-\infty}^{\infty} d p \frac{(\partial / \partial p)^{N-1} \check{f}(p, \boldsymbol{\xi})}{p-\boldsymbol{\xi} \cdot \mathbf{x}}
$$

if $N$ is even and

$$
f(\mathbf{x})=\frac{1}{2(2 \pi i)^{N-1}} \int_{|\boldsymbol{\xi}|=1}\left(\frac{\partial}{\partial p}\right)^{N-1} \check{f}(\boldsymbol{\xi} \cdot \mathbf{x}, \boldsymbol{\xi}) d \boldsymbol{\xi}
$$

when $N$ is odd, where $\partial \check{f}(\boldsymbol{\xi} \cdot \mathbf{x}, \boldsymbol{\xi}) / \partial p=[\partial \check{f}(p, \boldsymbol{\xi}) / \partial p]_{p=\boldsymbol{\xi} \cdot \mathbf{x}}$. Therefore, we can talk about the inverse Radon transform $\mathscr{R}^{-1}$. Thus $\mathscr{R}^{-1} \breve{f}=f$.

\section{THE RADON TRANSFORM OF INTEGRABLE AND RAPIDLY DECREASING BOEHMIANS}

In this section we define the Radon transform of an integrable Boehmian and discuss some of its properties. First we need some additional properties of the Radon transform.

Lemma 1. If $\left\{\phi_{n}(\mathbf{x})\right\} \in \Delta_{0}$, then $\left\{\check{\phi}_{n}(p, \xi)\right\} \in \Delta_{\Sigma}$.

Proof. It is easy to see from (3.2) that $\check{\phi}_{n}(p, \xi)=0$ if $p>s\left(\phi_{n}\right)$. Hence, for every $\xi \in S^{N-1}, s\left(\check{\phi}_{n}(\cdot, \xi)\right) \leq s\left(\phi_{n}\right) \rightarrow 0$. From (3.2) we have

$$
\check{\phi}_{n}(p, \boldsymbol{\xi})=\int_{p=\mathbf{x} \cdot \boldsymbol{\xi}} \phi_{n}(\mathbf{x}) d s,
$$

where $d s$ is the unit surface area on the hyperplane $p=\mathbf{x} \cdot \boldsymbol{\xi}$. Thus, for every $\boldsymbol{\xi} \in S^{N-1}$,

$$
\int_{\mathbb{R}} \check{\phi}_{n}(p, \boldsymbol{\xi}) d p=\int_{\mathbb{R}} \int_{p=\mathbf{x} \cdot \boldsymbol{\xi}} \phi_{n}(\mathbf{x}) d s d p=\int_{\mathbb{R}^{N}} \phi_{n}(\mathbf{x}) d \mathbf{x}=1 .
$$

Similarly,

$$
\int_{\mathbb{R}}\left|\check{\phi}_{n}(p, \boldsymbol{\xi})\right| d p \leq \int_{\mathbb{R}} \int_{p=\mathbf{x} \cdot \boldsymbol{\xi}}\left|\phi_{n}(\mathbf{x})\right| d s d p=\int_{\mathbb{R}^{N}}\left|\phi_{n}(\mathbf{x})\right| d \mathbf{x} \leq M .
$$

From (4.2) and (4.3) it follows that

$$
\int_{\|\boldsymbol{\xi}\|=1} \int_{\mathbb{R}} \check{\phi}_{n}(p, \boldsymbol{\xi}) d p d \boldsymbol{\xi}=A_{N-1}
$$


and

$$
\int_{\|\boldsymbol{\xi}\|=1} \int_{\mathbb{R}}\left|\check{\phi}_{n}(p, \boldsymbol{\xi})\right| d p d \boldsymbol{\xi} \leq M A_{N-1}
$$

where $A_{N-1}$ is the surface area of $S^{N-1}$. It also follows from (4.3) that if $f(\mathbf{x})$ is integrable over $\mathbb{R}^{N}$, then $\check{f}(p, \xi)$ is integrable for every $\xi \in S^{N-1}$; that is, if $f \in L^{1}\left(\mathbb{R}^{N}\right)$ then $\check{f}(\cdot, \xi) \in L^{1}(\mathbb{R})$.

The following property of the Radon transform is essential to our work [2, p. 95]: If $f$ and $g$ are integrable functions on $\mathbb{R}^{N}$, then

$$
(f * g)^{\vee}=\check{f} \odot \check{g} .
$$

Lemma 2. If $F=\left[f_{n} / \phi_{n}\right] \in \mathfrak{B}_{\mathscr{L}}$, then $\left[\check{f}_{n} / \check{\phi}_{n}\right] \in \mathfrak{B}_{\mathscr{L}(\Sigma)}$.

Proof. From Lemma 1 we know that for every $\boldsymbol{\xi} \in S^{N-1},\left\{\check{\phi}_{n}(\cdot, \xi)\right\}$ is a delta sequence. Moreover, by (4.4), $\check{f}_{n} \odot \check{\phi}_{m}=\left(f_{n} * \phi_{m}\right)^{\vee}=\left(f_{m} * \phi_{n}\right)^{\vee}=\check{f}_{m} \odot \check{\phi}_{n}$.

The above lemma justifies

Definition 1. The Radon transform $\mathscr{R} F=\check{F}$ of a Boehmian $F=\left[f_{n} / \phi_{n}\right] \in$ $\mathfrak{B}_{\mathscr{L}}$ is the Boehmian $\check{F}=\left[\check{f}_{n} / \check{\phi}_{n}\right] \in \mathfrak{B}_{\mathscr{L}(\Sigma)}$.

As mentioned in $\S 2, \mathscr{S}\left(\mathbb{R}^{N}\right)$ can be identified with a subspace of $\mathfrak{B}_{\mathscr{S}}$ and $\mathscr{S}\left(\Sigma^{N}\right)$ with a subspace of $\mathfrak{B}_{\mathscr{S}(\Sigma)}$. Let $\mathscr{I}_{\mathscr{S}}: \mathscr{S}\left(\mathbb{R}^{N}\right) \rightarrow \mathfrak{B}_{\mathscr{S}}$ be defined by $\mathscr{I}_{\mathscr{S}}(f)=\left[\left(f * \delta_{n}\right) / \delta_{n}\right]$ where $\left\{\delta_{n}\right\} \in \Delta_{0}$. Similarly, let $\mathscr{I}_{\Sigma}: \mathscr{S}\left(\Sigma^{N}\right) \rightarrow \mathfrak{B}_{\mathscr{S}(\Sigma)}$ be defined by $\mathscr{I}_{\Sigma}(f)=\left[\left(f \odot \phi_{n}\right) / \phi_{n}\right]$ where $\left\{\phi_{n}\right\} \in \Delta_{\Sigma}$. We have the following simple, but important theorem.

Theorem 1. $\mathscr{I}_{\Sigma} \mathscr{R}=\mathscr{R} \mathscr{I}_{\mathscr{S}}$.

In other words, the following diagram commutes:

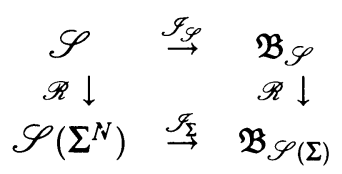

Proof. Let $f \in \mathscr{S}$ and $\left\{\delta_{n}\right\} \in \Delta_{0}$, and let $\phi_{n}=\check{\delta}_{n}$. Then $\left\{\phi_{n}\right\} \in \Delta_{\Sigma}$ by Lemma 1 and

$$
\begin{aligned}
\mathscr{I}_{\Sigma} \mathscr{R}(f) & =\mathscr{I}_{\Sigma}(\check{f})=\left[\frac{\check{f} \odot \phi_{n}}{\phi_{n}}\right] \\
& =\left[\frac{\check{f} \odot \check{\delta}_{n}}{\check{\delta}_{n}}\right]=\left[\frac{\left(f * \delta_{n}\right)^{\vee}}{\check{\delta}_{n}}\right]=\mathscr{R}\left[\frac{f * \delta_{n}}{\delta_{n}}\right]=\mathscr{R} \mathscr{I}_{\mathscr{S}}(f) .
\end{aligned}
$$

Theorem 2. The Radon transform $\mathscr{R}: \mathfrak{B}_{\mathscr{S}} \rightarrow \mathfrak{B}_{\Sigma}$ is sequentially continuous, i.e., if $\Delta-\lim _{n \rightarrow \infty} F_{n}=F$ in $\mathfrak{B}_{\mathscr{S}}$, then $\Delta-\lim _{n \rightarrow \infty} \mathscr{R}\left(F_{n}\right)=\mathscr{R}(F)$ in $\mathfrak{B}_{\Sigma}$.

Proof. Let $\Delta-\lim _{n \rightarrow \infty} F_{n}=F$ in $\mathfrak{B}_{\mathscr{S}}$. Then there exists a delta sequence $\left\{\delta_{n}\right\} \in \Delta_{0}$ such that $\lim _{n \rightarrow \infty}\left(F_{n}-F\right) * \delta_{n}=0$ in $\mathscr{S}\left(\mathbb{R}^{N}\right)$. Since the Radon transform is continuous, which follows from (3.7), we have

$$
\lim _{n \rightarrow \infty} \mathscr{R}\left(\left(F_{n}-F\right) * \delta_{n}\right)=\lim _{n \rightarrow \infty}\left(\check{F}_{n}-\check{F}\right) \odot \check{\delta}_{n}=0 .
$$

Since $\left\{\check{\delta}_{n}\right\} \in \Delta_{\Sigma}$, the above means that $\Delta-\lim _{n \rightarrow \infty} \mathscr{R}\left(F_{n}\right)=\mathscr{R}(F)$ in $\mathfrak{B}_{\Sigma}$. 
Gelfand, Graev, and Vilenkin defined the Radon transform for tempered distributions on $\mathbb{R}^{N}$. A tempered distribution $f \in \mathscr{S}^{\prime}\left(\mathbb{R}^{N}\right)$ is a rapidly decreasing Boehmian if and only if $f * \phi \in \mathscr{S}\left(\mathbb{R}^{N}\right)$ for every $\phi \in \mathscr{D}\left(\mathbb{R}^{N}\right)$. More precisely, if $f \in \mathscr{S}^{\prime}\left(\mathbb{R}^{N}\right)$ and $f * \phi \in \mathscr{S}\left(\mathbb{R}^{N}\right)$ for every $\phi \in \mathscr{S}\left(\mathbb{R}^{N}\right)$, then $f$ can be identified with $\left[\left(f * \delta_{n}\right) / \delta_{n}\right] \in \mathfrak{B}_{\mathscr{S}}$, where $\left\{\delta_{n}\right\} \in \Delta_{0}$. It is easy to show that this identification is independent of the choice of a particular delta sequence $\left\{\delta_{n}\right\} \in \Delta_{0}$.

On the other hand, the Radon transform of a tempered distribution $f$ such that $f * \phi \in \mathscr{S}\left(\mathbb{R}^{N}\right)$ for every $\phi \in \mathscr{D}\left(\mathbb{R}^{N}\right)$ can be identified with $\left[\check{f} \odot \check{\delta}_{n} / \check{\delta}_{n}\right]$, where $\left\{\delta_{n}\right\} \in \Delta_{0}$ and $\check{f} \odot \check{\delta}_{n}=\left(f * \delta_{n}\right)^{\vee}$. Again, it is easy to show that this identification is independent of the choice of a particular delta sequence $\left\{\delta_{n}\right\} \in \Delta_{0}$. Since

$$
\left(f * \delta_{n}\right)^{\vee} \odot \check{\delta}_{m}=\left(\left(f * \delta_{n}\right) * \delta_{m}\right)^{\vee}=\left(\left(f * \delta_{m}\right) * \delta_{n}\right)^{\vee}=\left(f * \delta_{m}\right)^{\vee} \odot \check{\delta}_{n}
$$

and $\left(f * \delta_{n}\right)^{\vee} \in \mathscr{S}\left(\Sigma^{N}\right)$, it follows that $\left[\left(f * \delta_{n}\right)^{\vee} / \check{\delta}_{n}\right] \in \mathfrak{B}_{\mathscr{S}(\Sigma)}$. Moreover, Theorem 1 remains true, i.e., the following diagram commutes:

$$
\begin{gathered}
\mathscr{S}^{\prime} \rightarrow \mathfrak{B}_{\mathscr{S}} \\
\mathscr{R} \downarrow \\
\mathscr{\mathscr { S }}_{\Sigma}^{\prime} \rightarrow \mathfrak{R}_{\mathscr{S}(\Sigma)}
\end{gathered}
$$

where the horizontal arrows denote the above described imbeddings. The result follows directly from the given definitions.

Clearly, there are tempered distributions which are not rapidly decreasing Boehmians. On the other hand, there are rapidly decreasing Boehmians which are not distributions. We now give an example of such a Boehmian.

Let $F=\left[f_{n} / \delta_{n}\right]$ be a rapidly decreasing Boehmian. If $f_{n} \in \mathscr{D}$ for all $n \in \mathbb{N}$, then $F$ is called a Boehmian with compact support. (The notion of support can be defined for Boehmians and it can be proved that $F=\left[f_{n} / \delta_{n}\right]$ has compact support if and only if $f_{n} \in \mathscr{D}$ for all $n \in \mathbb{N}$, see [9].) We will construct a Boehmian with compact support of infinite order. Obviously, such a Boehmian is not a distribution. Our construction is a modification of the example given by Boehme in [1].

From the Denjoy-Carleman theorem it follows that there exists $\psi \in \mathscr{D}(\mathbb{R})$ such that $\int \psi=1$ and

$$
\left|\psi^{(n)}(t)\right| \leq A B^{n}(2 n) ! \quad \text { for all } t \in \mathbb{R} .
$$

Define $\phi(\mathbf{x})=\psi\left(x_{1}\right) \cdots \psi\left(x_{N}\right), \phi_{n}(\mathbf{x})=n^{N} \phi(n \mathbf{x})$, and

$$
f_{n}(\mathbf{x})=\sum_{k=0}^{\infty} \frac{\partial^{k} \phi_{n}(\mathbf{x}) / \partial x_{1}^{k}}{(3 k) !} .
$$

Then $F=\left[f_{n} / \phi_{n}\right]$ is a Boehmian with compact support, thus a rapidly decreasing Boehmian. To calculate the Radon transform of $F$ we use

Lemma 3. Let $\phi_{n}(\mathbf{x})=n^{N} \phi(n \mathbf{x})$ for some function $\phi$ with compact support such that $\int_{\mathbf{R}^{N}} \phi(\mathbf{x}) d \mathbf{x}=1$. Then

$$
\mathscr{R}\left(\phi_{n}(\mathbf{x})\right)=n \check{\phi}(n p, \boldsymbol{\xi})
$$


and

$$
\mathscr{R}\left(\frac{\partial^{|k|} \phi_{n}(\mathbf{x})}{\partial x_{1}^{k_{1}} \cdots \partial x_{N}^{k_{N}}}\right)=n^{N-|k|}\left(\xi_{1}^{k_{1}} \cdots \xi_{N}^{k_{N}}\right) \frac{\partial^{|k|} \check{\phi}(n p, \boldsymbol{\xi})}{\partial p^{|k|}} .
$$

Proof. By (3.5) we have for $A=n I, \check{\phi}(n \mathbf{x})=n^{-N} \check{\phi}(p, \xi / n)$. Hence, by (3.4),

$$
\mathscr{R}\left(\phi_{n}(\mathbf{x})\right)=n^{N} \mathscr{R}(\phi(n \mathbf{x}))=\check{\phi}(p, \boldsymbol{\xi} / n)=\check{\phi}(n p / n, \boldsymbol{\xi} / n)=n \check{\phi}(n p, \boldsymbol{\xi}) .
$$

Then we prove (4.6) for $|k|=1=k_{1}$.

$$
\begin{aligned}
\frac{\partial \phi(n \mathbf{x})}{\partial x_{1}} & =\lim _{\varepsilon \rightarrow 0} \frac{\phi\left(n x_{1}+\varepsilon, n x_{2}, \ldots, n x_{N}\right)-\phi\left(n x_{1}, n x_{2}, \ldots, n x_{N}\right)}{\varepsilon} \\
& =\lim _{\varepsilon \rightarrow 0} \frac{\phi\left(u_{1}+\varepsilon, u_{2}, \ldots, u_{N}\right)-\phi\left(u_{1}, u_{2}, \ldots, u_{N}\right)}{\varepsilon}
\end{aligned}
$$

where $u_{i}=n x_{i}, i=1, \ldots, N$. Since $\mathbf{x} \cdot \boldsymbol{\xi}=p$, we have $\mathbf{u} \cdot \boldsymbol{\xi}=q=n p$. Therefore, by taking the Radon transform of both sides of (4.7) and using (3.6) with $\mathbf{a}=(\varepsilon, 0, \ldots, 0)$, we obtain

$$
\mathscr{R}\left(\frac{\partial \phi(n \mathbf{x})}{\partial x_{1}}\right)=\lim _{\varepsilon \rightarrow 0} \frac{\check{\phi}\left(q+\varepsilon \xi_{1}, \boldsymbol{\xi}\right)-\check{\phi}(q, \boldsymbol{\xi})}{\varepsilon}=\xi_{1} \frac{\partial \check{\phi}(q, \boldsymbol{\xi})}{\partial q}=\frac{\xi_{1}}{n} \frac{\partial \check{\phi}(n p, \boldsymbol{\xi})}{\partial p} \text {. }
$$

Thus, by repeated application of the above, we obtain (4.6).

Now we can find the Radon transform of $F$ :

$$
\begin{aligned}
\mathscr{R}(F) & =\mathscr{R}\left[\frac{\sum_{k=0}^{\infty}\left[\left(\partial^{k} / \partial x_{1}^{k}\right) \phi_{n}(\mathbf{x}) /(3 k) !\right]}{\phi_{n}(\mathbf{x})}\right] \\
& =\left[\frac{\sum_{k=0}^{\infty}\left[n^{N-k} \xi_{1}^{k}\left(\partial^{k} / \partial p^{k}\right) \check{\phi}(n p, \boldsymbol{\xi}) /(3 k) !\right]}{n \check{\phi}(n p, \boldsymbol{\xi})}\right] .
\end{aligned}
$$

The inversion formulae for the Radon transform of rapidly decreasing functions given in $\S 3$ can be easily generalized to Radon transforms of rapidly decreasing Boehmians. Let $\check{F} \in \mathfrak{B}_{\mathscr{S}(\Sigma)}$ be the Radon transform of a rapidly decreasing Boehmian $F$, i.e., $\check{F}=\left[\check{f}_{n} / \check{\delta}_{n}\right]$ for some $f_{n} \in \mathscr{S}\left(\mathbb{R}^{N}\right)$ and $\left\{\delta_{n}\right\} \in \Delta_{0}$. Then

$$
\mathscr{R}^{-1}(\check{F})=\mathscr{R}^{-1}\left[\check{f}_{n} / \check{\delta}_{n}\right]=\left[\mathscr{R}^{-1}\left(\check{f}_{n}\right) / \mathscr{R}^{-1}\left(\check{\delta}_{n}\right)\right]=\left[f_{n} / \delta_{n}\right]=F
$$

\section{REFERENCES}

1. T. K. Boehme, The support of Mikusinski operators, Trans. Amer. Math. Soc. 176 (1973), 319-334.

2. S. Deans, The Radon transform and some of its applications, Wiley, New York, 1983.

3. I. M. Gelfand and G. E. Shilov, Generalized functions, Vols. 1 \& 2, Academic Press, New York, 1964.

4. I. M. Gelfand, M. I. Graev, and N. Ya. Vilenkin, Generalized functions, Vol. 5, Academic Press, New York, 1966.

5. S. Helgason, The Radon transform, Birkhäuser, Boston-Basel-Stuttgart, 1980.

6. D. Ludwig, The Radon transform on Euclidean spaces, Comm. Pure Appl. Math. 19 (1966), 49-81.

7. J. Mikusiński, Operational calculus, Pergamon Press, London, 1959. 
8. J. Mikusiński and P. Mikusiński, Quotients de suites et leurs applications dans l'analyse fonctionnelle, C. R. Acad. Sci. Paris Sér. I Math. 293 (1981), 463-464.

9. P. Mikusiński, Convergence of Boehmians, Japan. J. Math. 9 (1983), 159-179.

10. __ Boehmians and generalized functions, Acta Math. Hungar. 51 (1988), 271-281.

11. J. Radon, Über die Bestimmung von Funktionen durch ihre Integralwärte längs gewisser Manningfaltigkeiten, Ber. Verh. Sächs. Akad. 69 (1917), 262-277.

12. W. Rudin, Real and complex analysis, 2nd ed., McGraw-Hill, New York, 1974.

13. L. Schwartz, Théorie des distributions, Hermann, Paris, 1950-1951.

14. A. H. Zemanian, Generalized integral transformations, Dover, New York, 1987.

Department of Mathematics, University of Central Florida, Orlando, Florida 32816 E-mail address, A. Zayed: FDZAYED@UCF1VM.Bitnet 